\title{
Ridge Preservation Using an Innovative Enzyme-deantigenic Equine Bone Paste: A Case Report with 36-month Follow-up
}

\author{
Danilo A Di Stefano ${ }^{1}$, Paolo Arosio $^{2}$, Lorenzo Cinci $^{3}$, Laura Pieri ${ }^{4}$
}

\begin{abstract}
Aim: This study aimed to report a successful clinical, histological, and histomorphometric outcome of a novel equine-derived bone paste for a ridge preservation surgery involving a single post-extractive socket.

Background: After tooth avulsion, unless the implant position is not carried out straightforwardly, the alveolar process undergoes resorption: to limit it, post-extractive sockets may be grafted according to the ridge preservation principles. Grafting materials should display proper biological properties and optimal handling characteristics. Bone pastes may facilitate grafting operations, avoid granules' dispersion, and maximize the contact of the graft with the surrounding bone. An innovative equine-derived bone paste has been recently introduced on the market, but its use has never been documented in the medical literature.

Case description: This report describes the treatment of a patient who received the equine-derived bone paste in a post-extractive socket to allow the preservation of the alveolar ridge and was later rehabilitated with a crown supported by a single implant.

Conclusion: The handling properties of the equine-derived bone paste were excellent. At the 36-month follow-up, the peri-implant bone levels had been maintained, with the implant being successful according to the Albrektsson and Zarb criteria. Histologic outcome showed that the bone paste was fully biocompatible; histomorphometric analysis showed that a significant amount of newly formed bone could be observed in the grafted socket.

Clinical significance: Alveolar ridge preservation using bone grafts is a well-known approach, yet there is still no agreement about which bone graft might be considered the most suitable for this indication. The novel equine-derived bone paste used in the present study appears a promising option for effective socket preservation and may promote secondary intention healing.

Keywords: Bone formation, Bone paste, Post-extractive sockets, Ridge preservation, Xenograft.

The Journal of Contemporary Dental Practice (2019): 10.5005/jp-journals-10024-2664
\end{abstract}

\section{BACKGROUND}

After tooth avulsion, the alveolar process undergoes a resorption following a spatial and temporal pattern that has been extensively studied. ${ }^{1,2}$ The ridge may resorb to such an extent that implant placement is no more feasible, or implant-supported rehabilitation would be at risk for a poor functional and esthetic outcome. ${ }^{2}$ Ridge contraction may be limited by grafting the post-extractive socket using a biomaterial, acting as a bone substitute, following the ridge preservation ${ }^{3}$ technique. While an autogenous bone is still being regarded as the gold standard graft, as it contains both living cells and growth factors, ${ }^{4}$ its use exposes the patient to additional intra- and post-surgical risks. ${ }^{5}$ For this reason, many synthetic or natural biomaterials have been proposed as an alternative. ${ }^{6}$ Xenografts (i.e., bone grafts manufactured by processing bone tissue from a non-human mammal) may in principle present a biological advantage over other natural or synthetic grafts because of the similarity in bone tridimensional structure and chemical composition humans shares with the other mammals. ${ }^{7}$ Among xenografts, recent studies have proposed an enzyme-deantigenic equine bone (EDEB) as an alternative to an organic bovine bone (ABB), with the xenograft having the longest history of use in oral surgery and maxillofacial surgery. ${ }^{8,9}$ Different from $A B B, E D E B$ is manufactured by subjecting a bovine bone to high temperatures, which eliminates all organic components. ${ }^{10}$ EDEB is made non-antigenic by subjecting an equine bone to the action of lytic enzymes and has already been used in different kinds of oral and maxillofacial surgery applications requiring bone grafting. ${ }^{11-18}$ It was used effectively also in orthopedic applications. ${ }^{19}$
${ }^{1}$ Dental School, Vita-Salute University and IRCCS San Raffaele, Milan, Italy

${ }^{2}$ Private Practitioner, Vimercate, Italy

${ }^{3}$ Department of Neuroscience, Psychology, Drug Research and Child Health (Neurofarba), Pharmacology and Toxicology Section, University of Florence, Florence, Italy

${ }^{4}$ Department of Health Sciences, University of Florence, Florence, Italy Corresponding Author: Danilo A Di Stefano, Dental School, VitaSalute University and IRCCS San Raffaele, Milan, Italy, Phone: +39 02 48705703, e-mail: distefano@centrocivitali.it

How to cite this article: Di Stefano DA, Arosio P, Cinci L, et al. Ridge Preservation Using an Innovative Enzyme-deantigenic Equine Bone Paste: A Case Report with 36-month Follow-up. J Contemp Dent Pract 2019;20(10):1229-1234.

Source of support: The work was partially sponsored by Bioteck S.p.A., which donated the bone paste. Data belonged to the authors and by no means did the manufacturer interfere with the conduct of the case or the publication of its results.

Conflict of interest: None

When biomaterials comprising xenografts are used for socket preservation, their handling characteristics may facilitate grafting, helping the surgeon to fill the socket easily and contributing to graft stabilization. Recently, a novel bone paste has been introduced on the market; it consists of a polyethylene glycol/hydroxyl-propyl methyl cellulose-based hydrogel (PEG/HPMC) that acts as a carrier, containing cancellous and cortical EDEB granules and 
equine-demineralized bone matrix. ${ }^{20}$ The gel is added with a subsidiary amount of vitamin $\mathrm{C}$ acting as a viscomodulator. Bone pastes allow avoiding granule dispersion and loss during surgery, and to assure complete filling and direct contact with the tissue surrounding the defect, possibly facilitating bone repair. It has been shown in an in vitro study on human bone marrow stem cells that this novel equine-derived bone paste stimulates the expression of a known modulator of bone regeneration as the RUNT-related transcription factor 2 (RUNX2), bone bialoprotein (BSP), and osteocalcin. ${ }^{20}$ The same study also investigated in vivo the effects of this bone paste when grafted in artificially induced femoral defects in rabbits. A histological analysis of the grafted sites at 1 and 2 months showed an interesting reorganization of the regenerating tissue within the lesion boundaries. ${ }^{20}$ While EDEB has already been used in oral, maxillofacial, and orthopedic surgeries, to the author's knowledge, no clinical application of this equine bone paste was ever reported in the literature. The aim of the present study is to illustrate the clinical, histological, and histomorphometric outcome relating to a clinical case where this novel bone paste was used successfully to carry out a socket preservation surgery.

\section{Case Description}

The patient was a 45-year-old man with a non-contributory medical history presenting with pain at mastication in his lower right quadrant. Clinical and radiographic examination revealed tooth 4.7, already devitalized and rehabilitated with a ceramic crown, presented with a large carious lesion and was definitively compromised (Fig. 1A). A two-step procedure that included grafting the socket to preserve it from resorption and consequent placement of an implant was developed with the aim of rehabilitating the patient with a single crown. The patient provided informed consent.

The patient underwent thorough oral hygiene 2 days before surgery. For antibiotic prophylaxis, $2 \mathrm{~g}$ of amoxicillin/clavulanic acid (Augmentin, Glaxo-SmithKline, Verona, Italy) was administered one hour before surgery and then every 12 hours for eight days. The patient also rinsed for 2 minutes with chlorhexidine $0.20 \%$ mouth rinse (Corsodyl, Glaxo-SmithKline) and received $100 \mathrm{mg}$ of a nonsteroidal-anti-inflammatory drug (Aulin, Roche, Milano, Italy). Local anesthetic was administered by means of infiltration into the oral mucosa with $1 \%$ articaine with epinephrine 1:100,000 (Molteni Dental, Milano, Italy).

The tooth was extracted atraumatically (Figs $1 \mathrm{~B}$ and C) and the socket debrided from any residual of fibrous tissue. Subsequently, the bone paste (Activabone ${ }^{\circledR}$ Mouldable Paste, Bioteck, Arcugnano, Italy, details provided in the following paragraph) was extruded from its syringe directly over the socket (Fig. 1D) and gently pressed with a round instrument to fill the socket (Fig. 1E). No flaps were elevated. After grafting, cross stitches (5-0 non-resorbable suture (Monomyd, Butterfly, Cavenago, Italy)) were applied to stabilize the graft (Fig. 1F). Gingival rims were left open, seeking for second intention healing. Second surgery followed after 3 months. The clinical appearance of soft tissues was quite satisfying (Fig. 2A) as well as the radiographic appearance of the grafted area (Fig. 2B). Antibiotic prophylaxis and post-surgical treatment, anesthetic treatment, and pain management were carried out as in the first intervention. After gaining access to the bone ridge, a bone core (Figs $2 \mathrm{C}$ to $\mathrm{E}$ ) was collected using a trephine and placed in a test tube containing buffered $10 \%$ formalin for subsequent histological analysis. The implant site was then prepared following the drilling
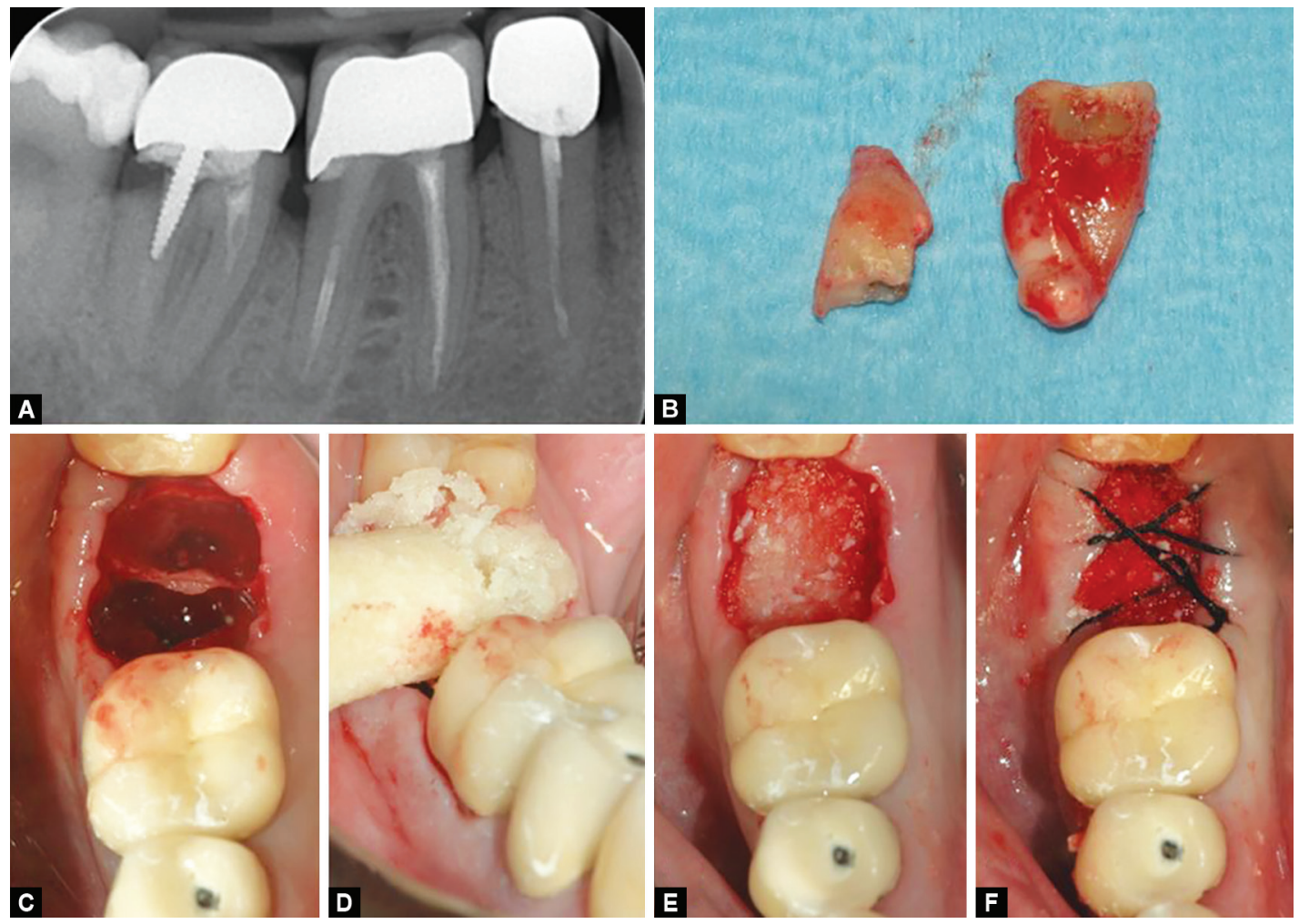

Figs $1 \mathrm{~A}$ to F:Tooth 4.7 (A), already devitalized, presents a large carious lesion and, being compromised, has been extracted (B). The post extractive socket $(C)$ is filled with the equine bone paste that is extruded directly into the socket (D) and pressed gently with a round instrument to fill the socket (E). No membrane is placed and the gingival rims are stabilized with a cross stitch (F) 

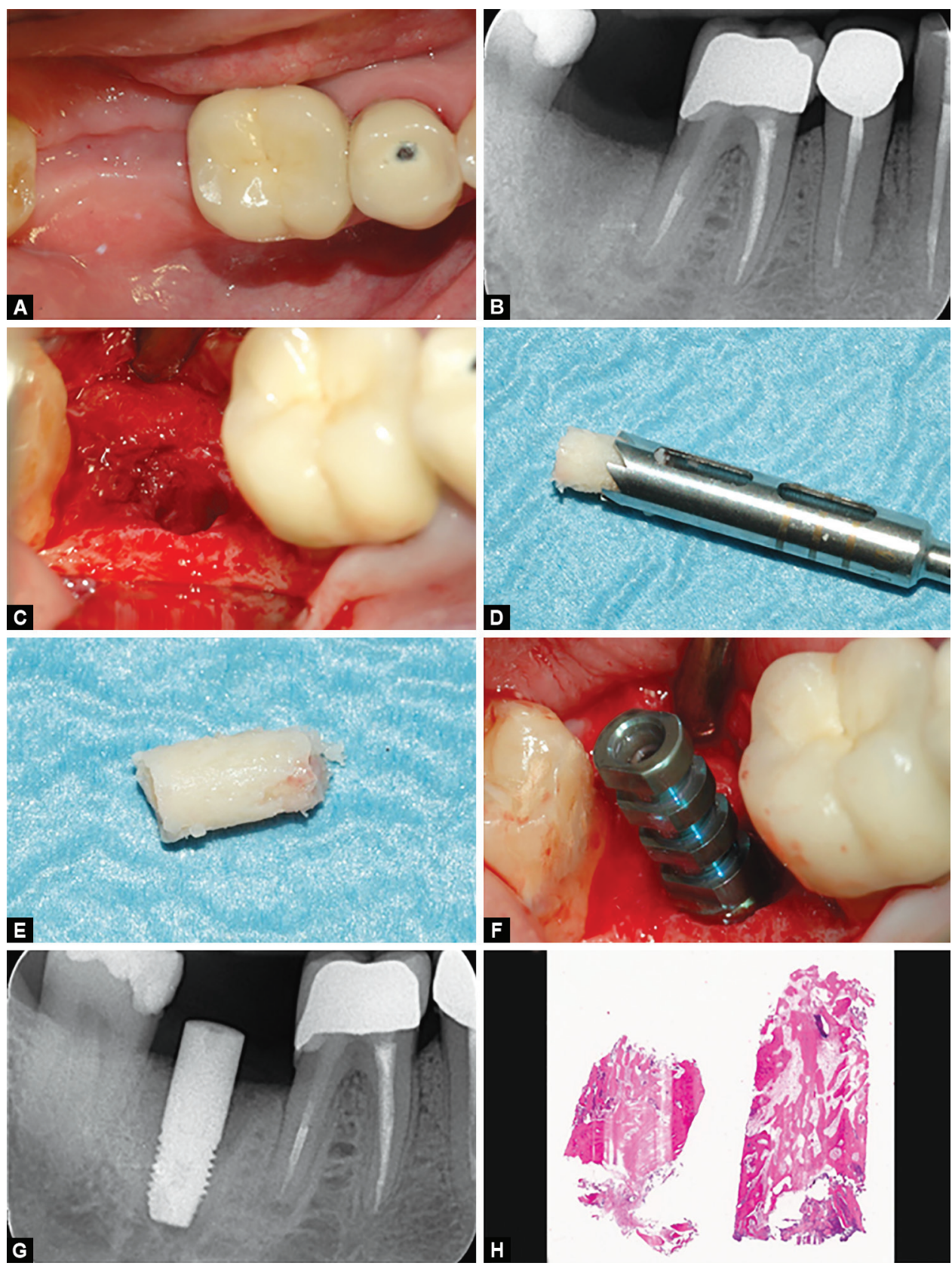

Figs $2 \mathrm{~A}$ to $\mathrm{H}$ : (A) The clinical appearance of soft tissue after three months from the first surgery was satisfactory as well as (B) the radiographic appearance of the grafted area. Before placing the fixture, a bone core ( $\mathrm{C}$ to $\mathrm{E})$ was collected in correspondence of the grafted area. An implant was then positioned, leaving it non-submerged ( $\mathrm{F}$ and $\mathrm{G}$ ). The histologic analysis ( $\mathrm{H}$; hematoxlylin-eosin, $3.5 \times)$ shows that the bone paste was fully biocompatible, and allowed a significant bone regeneration

sequence advised by the manufacturer and a $5 \times 10 \mathrm{~mm}$ cylindrical implant (Stone, IDI Evolution, Concorezzo, Italy) was positioned and was left non-submerged (Figs $2 \mathrm{~F}$ and $\mathrm{G}$ ). A provisional crown was delivered after 3.5 months; definitive crown delivery followed after 1 month. The patient was followed up every month for the following 6 months, and then every 6 months up to 36 months after implant surgery.

\section{Bone Paste}

The innovative equine-derived bone paste used in the present study (Activabone ${ }^{\circledR}$ Mouldable Paste, Bioteck, Arcugnano, Italy) contains equine bone and equine demineralized bone matrix
(DBM) and a hydrogel as a carrier. Equine bone and DBM are achieved through Zymo-Teck ${ }^{\circledR}$ and the Bioteck proprietary enzymatic deantigenation process, which guarantees grafts with preserved biological and biomechanical properties. This processing is performed at physiological temperature $\left(37^{\circ} \mathrm{C}\right)$, and removes completely the antigens from the bone tissue, without affecting the native quaternary structure of bone collagen and extracellular matrix components, which are therefore totally preserved. Equine bone and DBM components in the Activabone ${ }^{\circledast}$ paste are: type I bone collagen of equine origin (DBM); equine cancellous bone micro-granules, equine cancellous, and cortical (1:1) bone granules having a $0.5-1 \mathrm{~mm}$ diameter. The carrier is a low-molecular-weight 
polymeric hydrogel, consisting in a mixture of water and poly ethylene glycol/hydroxyl-propyl methyl cellulose-based hydrogel (PEG/HPMC). The mixture is added with a subsidiary amount of vitamin $\mathrm{C}$, acting as a viscomodulator agent (patented), as vitamin $C$ is able to limit intra- and inter-molecular rearrangement of PEG and HPMC polymeric chains originating as a consequence of sterilization, thus maintaining the visco-elasticity of gels and injectability of bone fillers nearly unaltered. ${ }^{20}$ The paste undergoes beta-sterilization at $25 \mathrm{kGy}$, and is provided to the oral surgeon in sterile-packaged syringes.

\section{Histological and Histomorphometric Analysis}

The tube containing the biopsy was marked with an alphanumeric code and sent to the histologists (LC, LP) who were, therefore, unaware of the material used for grafting. Bone cores were decalcified for 21 days in a $0.76 \mathrm{M}$ sodium formate and $1.6 \mathrm{M}$ formic acid solution (Panreac Quimica, Barcelona, Spain). The sample was subsequently dehydrated in ascending concentrations of ethanol and embedded in paraffin. This procedure aimed at achieving rapid tissue infiltration with only minimal sample shrinkage, in order to provide a sample morphology still highly representative of the in vivo bone features. The bone core was cut into 5 - $\mu$ m-thick sections, mounted on slides, and stained with hematoxylin-eosin. One assessor (LC) provided a qualitative report aimed at identifying any sign of inflammatory or immune reactions and provided a general qualitative assessment. Slides were also observed under a polarized light to assess the presence of lamellar (mature), mineralized bone. Morphometrical measurements were performed on digital photomicrographs collected at $10 \times$ magnification. The whole sample image was analyzed independently by two of the authors (LC, LP) using the Image J 1.33 analysis software (National Institute of Health, Bethesda, USA). The total sample area (TSA), the total bone area (TBA), the newly formed bone area (LBA), and the residual bone substitute area (RBA) were measured. Each of the two assessors repeated the assessment in triplicate. Average newly formed bone (NFB) and residual biomaterial (RB) were then calculated and expressed as the percentage over the total sample area $(\% N F B=L B A \times 100 / T S A ; \% R B=R B A \times 100 / T S A)$.

\section{Results}

The patient healed uneventfully and the final appearance of the rehabilitation was still quite satisfactory (Fig. 3), with no gingival recession evident. Bone levels were maintained between the grafting procedure and implant placement, confirming the socket preservation grafting surgery worked effectively. Bone peri-implant levels were found to be maintained (Fig. 3E) also at the following controls. At the 36-month follow-up, the prosthetic crown was working effectively and the implant met the requirements for success defined by Albrektsson et al. ${ }^{21}$ Qualitative histologic analysis showed a quite extended newly-formed bone area throughout all the sample (Fig. 2H). A small number of residual bone graft particles could be observed because of their affinity for
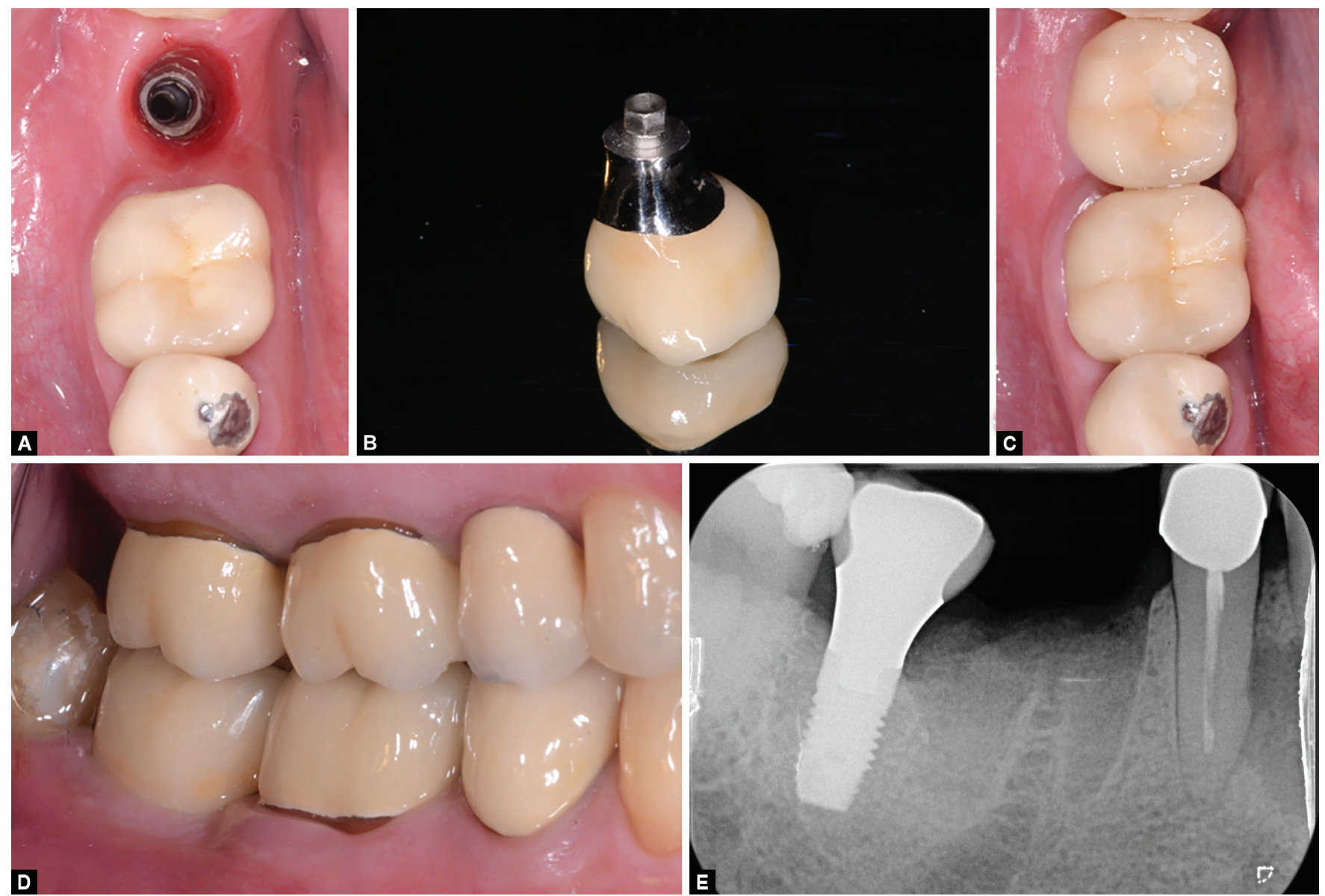

Figs $3 \mathrm{~A}$ to $\mathrm{E}$ : (A) After proper soft tissue conditioning; (B) A metal-ceramic crown is fabricated; (C and D) Delivered to the patient; (E) At the 36-month follow-up, peri-implant levels satisfy the success criteria of Albrektsson and Zarb and the prosthesis is fully functional 
hematoxylin and for having bone lacunae devoid of osteocytes. Residual particles were in close contact with the newly formed bone. No signs of inflammation or cartilage-like tissue could be observed, confirming all the components of the bone paste are fully biocompatible. Lamellar (mature) mineralized bone areas could be observed in most of the sample. Histomorphometric results were $\mathrm{NFB}=60.12 \%$ and $\mathrm{RB}=20.53 \%$.

\section{Discussion}

Ridge preservation involves using a bone graft that prevents, at least partially, bone resorption to occur; indeed, Vignoletti et al. ${ }^{22}$ concluded in 2012 that although some degree of bone modelling and remodeling will occur after tooth extraction, different ridge preservation procedures resulted in significantly less vertical and horizontal contraction of the alveolar bone crest. Yet, although the use of barrier membranes, flap surgical procedure, and full flap closure demonstrated better results, no conclusion could be reached regarding the type of surgical procedure or the most suitable biomaterial. A similar conclusion was reached in 2015 by the Cochrane group, ${ }^{23}$ which stated on the basis of their meta-analysis of eight RCTs with a total of 233 extraction sites in 184 participants that there was still no convincing evidence of any clinically significant difference between different grafting materials and barriers used for ridge preservation and, accordingly, that further long term, well-designed RCTs were necessary. The matter is still debated, with investigations trying to determine the most effective graft also resorting to complex statistic meta-analysis models. ${ }^{24}$ The present case illustrates the successful use of a novel equine-derived bone paste; results were satisfying both considering the short-term clinical outcome and the histomorphometric data showing, at 4 months from the grafting surgery, a significant bone formation inside the socket. No inflammatory reactions could be observed, suggesting a neutral interaction of the bone paste with the newly regenerating bone tissue. This is consistent with previous histological and immunohistochemistry studies on EDEB in bone regeneration in other oral surgery applications. ${ }^{14,25,26}$ The significant amount of NFB observed may partially be explained by the behavior of osteoclasts that was observed when they were cultured on EDEB in previous in vitro experiments. ${ }^{27}$ This behavior may, in turn, be explained by the presence of native collagen in EDEB. ${ }^{13,14,23,27}$ Bone collagen is involved in or modulates many processes related to bone regeneration. ${ }^{28-31}$ Indeed, a randomized clinical trial on forty patients that compared the use of EDEB and anorganic bovine bone (ABB), not containing any collagen, ${ }^{10}$ for maxillary sinus augmentation, showed that grafting EDEB resulted in a greater quantity of newly formed bone (NFB), and a smaller quantity of residual biomaterial (RB) at implant insertion than when $A B B$ was used, ${ }^{25}$ and a retrospective study investigated bone formation over time following maxillary sinus augmentation using EDEB and showed that new bone formation occurred at an early time ( $<3$ months) after grafting. ${ }^{26}$ Both results were explained by the claim that EDEB contains type I bone collagen in its native state.

Further, it may be speculated that vitamin $C$ within the hydrogel carrier may sustain collagen deposition throughout the entire lesion volume, vitamin $C$ being a requested co-factor for the activity of prolyl- and lysil-hydroxylases, essential enzymes for collagen fibril assembly. ${ }^{32}$ An additional advantage of a bone paste with respect to a granular graft is the carrier providing spatial stabilization to granules while at the same time facilitating the contact of the graft with all the surrounding vital bone. The authors appreciated, in particular, the handling properties of the equine bone paste that was firm but, at the same time, easily moldable and could be easily adapted to the socket. Finally, it should be remarked that the positive results experienced in this clinical case were achieved without using any membrane and leaving the socket to heal by second intention.

\section{Conclusion}

The equine-derived bone paste used in the present case allowed for successful socket preservation, and, taking into consideration its handling properties as well, it might be a promising bone graft to carry out ridge preservation surgeries. Accordingly, its actual effectiveness for this indication should be the subject of further investigations.

\section{Clinical Significance}

Alveolar ridge preservation using bone grafts is a well-known approach, yet there is still no agreement about which bone graft might be considered the most suitable for this indication. The novel equine-derived bone paste used in the present study appears a promising option for effective socket preservation and may promote secondary intention healing.

\section{ACKNOWLedgment}

Danilo Alessio Di Stefano has a scientific consultancy relationship with Bioteck S.p.A. All other authors declare that they have no conflicts of interest.

\section{References}

1. Van der Weijden F, Dell'Acqua F, et al. Alveolar bone dimensional changes of post-extraction sockets in humans: a systematic review. J Clin Periodontol 2009 Dec;36(12):1048-1058. DOI: 10.1111/j.1600051X.2009.01482.x.

2. Araújo MG, Lindhe J. Ridge alterations following tooth extraction with and without flap elevation: an experimental study in the dog. Clin Oral Implants Res 2009 Jun;20(6):545-549. DOI: 10.1111/j.16000501.2008.01703.x.

3. De Risi V, Clementini $M$, et al. Alveolar ridge preservation techniques: a systematic review and meta-analysis of histological and histomorphometrical data. Clin Oral Implants Res 2015 Jan;26(1): 50-68. DOI: $10.1111 / \mathrm{clr}$.12288.

4. Misch CM. Maxillary autogenous bone grafting. Oral Maxillofac Surg Clin North Am 2011 May;23(2):229-238. DOI: 10.1016/j.coms. 2011.01.003.

5. Nkenke $E$, Weisbach $V$, et al. Morbidity of harvesting of bone grafts from the iliac crest for preprosthetic augmentation procedures: a prospective study. Int J Oral Maxillofac Surg 2004 Mar;33(2):157-163. DOI: 10.1054/ijom.2003.0465.

6. Esposito M, Grusovin MG, et al. Interventions for replacing missing teeth: horizontal and vertical bone augmentation techniques for dental implant treatment. Cochrane Database Syst Rev 2009 Oct 7(4):CD003607. DOI: 10.1002/14651858.CD003607.pub4.

7. Aerssens J, Boonen S, et al. Interspecies differences in bone composition, density, and quality: potential implications for in vivo bone research. Endocrinology 1998 Feb;139(2):663-670.DOI: 10.1210/ endo.139.2.5751.

8. Baldini N, De Sanctis $M$, et al. Deproteinized bovine bone in periodontal and implant surgery. Dent Mater 2011 Jan;27(1):61-70. DOI: 10.1016/j.dental.2010.10.017.

9. Jensen SS, Terheyden H. Bone augmentation procedures in localized defects in the alveolar ridge: clinical results with different bone grafts and bone-substitute materials. Int J Oral Maxillofac Implants 2009;24(Suppl):218-236. 
10. Benke $D$, Olah A, et al. Protein-chemical analysis of Bio-Oss bone substitute and evidence on its carbonate content. Biomaterials 2001 May;22(9):1005-1012. DOI: 10.1016/S0142-9612(00)00323-9.

11. Felice $P$, Piana $L$, et al. Vertical ridge augmentation of an atrophic posterior mandible with an inlay technique and cancellous equine bone block: a case report. Int J Periodontics Restorative Dent 2013 Mar-Apr;33(2):159-166. DOI: 10.11607/prd.1098.

12. Pistilli $R$, Signorini $L$, et al. Case of severe bone atrophy of the posterior maxilla rehabilitated with blocks of equine origin bone: histological results. Implant Dent 2013 Feb;22(1):8-15. DOI: 10.1097/ ID.0b013e3182777239.

13. Di Stefano DA, Andreasi Bassi M, et al. Treatment of a bone defect consequent to the removal of a periapical cyst with equine bone and equine membranes: clinical and histological outcome. Minerva Stomatol 2012 Nov-Dec;61(11-12):477-490.

14. Artese L, Piattelli A, et al. Sinus lift with autologous bone alone or in addition to equine bone: an immunohistochemical study in man. Implant Dent 2011 Oct;20(5):383-388. DOI: 10.1097/ ID.0b013e3182310b3d.

15. De Angelis N, Scivetti M. Lateral ridge augmentation using an equine flex bone block infused with recombinant human platelet-derived growth factor BB: a clinical and histologic study. Int J Periodontics Restorative Dent 2011 Jul-Aug;31(4):383-388.

16. Ludovichetti $M, D i$ Stefano DA, et al. Vertical ridge augmentation using a flexible heterologous cortical bone sheet: three-year follow-up. Int J Periodontics Restorative Dent 2011 Jul-Aug;31(4): 401-407.

17. Di Stefano DA, Artese $L$, et al. Alveolar ridge regeneration with equine spongy bone: a clinical, histological, and immunohistochemical case series. Clin Implant Dent Relat Res 2009 Jun;11(2):90-100. DOI: 10.1111/j.1708-8208.2008.00104.x.

18. Stievano D, Di Stefano A, et al. Maxillary sinus lift through heterologous bone grafts and simultaneous acid-etched implants placement. Five year follow-up. Minerva Chir 2008 Apr;63(2):79-91.

19. Santini $S$, Barbera $P$, et al. Equine-derived bone substitutes in orthopedics and traumatology: authors' experience. Minerva Chir 2011 Feb;66(1):63-72.

20. Giannoni P, Villa F, et al. Rheological properties, biocompatibility and in vivo performance of new hydrogel-based bone fillers. Biomater Sci 2016 Nov 18;4(11):1691-1703. DOI: 10.1039/C6BM00478D.

21. Albrektsson T, Zarb G, et al. The long-term efficacy of currently used dental implants: a review and proposed criteria of success. Int J Oral Maxillofac Implants 1986;1(1):11-25.

22. Vignoletti $F$, Matesanz $P$, et al. Surgical protocols for ridge preservation after tooth extraction. A systematic review. Clin Oral Implants
Res 2012 Feb;23(Suppl 5):22-38. DOI: 10.1111/j.1600-0501.2011. 02331.x.

23. Atieh MA, Alsabeeha $\mathrm{NH}$, et al. Interventions for replacing missing teeth: alveolar ridge preservation techniques for dental implant site development. Cochrane Database Syst Rev 2015 May 28(5):CD010176. DOI: 10.1002/14651858.CD010176.pub2.

24. locca O, Farcomeni $A$, et al. Alveolar ridge preservation after tooth extraction: a Bayesian Network meta-analysis of grafting materials efficacy on prevention of bone height and width reduction. J Clin Periodontol 2017 Jan;44(1):104-114. DOI: 10.1111/jcpe. 12633.

25. Di Stefano DA, Gastaldi G, et al. Histomorphometric Comparison of Enzyme-Deantigenic Equine Bone and Anorganic Bovine Bone in Sinus Augmentation: A Randomized Clinical Trial with 3-Year Follow-Up. Int J Oral Maxillofac Implants 2015 Sept-Oct; 30(5):1161-1167. DOI: 10.11607/jomi.4057.

26. Di Stefano DA, Gastaldi G, et al. Bone Formation Following Sinus Augmentation with an Equine-Derived Bone Graft: A Retrospective Histologic and Histomorphometric Study with 36-Month Follow-up. Int J Oral Maxillofac Implants 2016 Mar-Apr;31(2):406-412. DOI: 10.11607/jomi.4373.

27. Perrotti V, Nicholls BM, et al. Human osteoclast formation and activity on an equine spongy bone substitute. Clin Oral Implants Res 2009 Jan;20(1):17-23. DOI: 10.1111/j.1600-0501.2008.01608.x.

28. Liu G, Hu YY, et al. Effect of type I collagen on the adhesion, proliferation, and osteoblastic gene expression of bone marrowderived mesenchymal stem cells. Chin J Traumatol 2004 Dec;7(6): 358-362.

29. Mizuno M, Fujisawa R, et al. Type I collagen-induced osteo-blastic differentiation of bone-marrow cells mediated by collagenalpha2beta1 integrin interaction. J Cell Physiol 2000 Aug; 184(2):207-213. DOI: 10.1002/1097-4652(200008)184:2<207::AID-JCP8 $>3.0 . \mathrm{CO} ; 2-\mathrm{U}$.

30. Regazzoni $\mathrm{C}$, Winterhalter $\mathrm{KH}$, et al. Type I collagen induces expression of bone morphogenetic protein receptor type II. Biochem Biophys Res Commun 2001 May 4;283(2):316-322. DOI: 10.1006/ bbrc.2001.4813.

31. Green J, Schotland S, et al. Cell-matrix interaction in bone: type I collagen modulates signal transduction in osteoblast-like cells. Am J Physiol 1995 May;268(5 Pt 1):C1090-C1103. DOI: 10.1152/ ajpcell.1995.268.5.C1090.

32. Grinnell F, Fukamizu H, et al. Collagen processing, crosslinking, and fibril bundle assembly in matrix produced by fibroblasts in longterm cultures supplemented with ascorbic acid. Exp Cell Res 1989 Apr;181(2):483-491. DOI: 10.1016/0014-4827(89)90105-5. 